Mathématiques et sciences humaines
Mathematics and social sciences

178 | Été 2007

Art, mathématiques, langage et émotion

\title{
Mathématiques, musique et émotion
}

Mathematics, music and emotion

Jean-Paul Allouche et Laurence Maillard-Teyssier

\section{OpenEdition}

Journals

Édition électronique

URL : http://journals.openedition.org/msh/4292

DOI : $10.4000 /$ msh.4292

ISSN : $1950-6821$

\section{Éditeur}

Centre d'analyse et de mathématique sociales de l'EHESS

\section{Édition imprimée}

Date de publication : 1 juillet 2007

Pagination : 119-124

ISSN : 0987-6936

Référence électronique

Jean-Paul Allouche et Laurence Maillard-Teyssier, « Mathématiques, musique et émotion »,

Mathématiques et sciences humaines [En ligne], 178 | Été 2007, mis en ligne le 21 septembre 2007 , consulté le 23 juillet 2020. URL : http://journals.openedition.org/msh/4292 ; DOI : https://doi.org/ $10.4000 /$ msh.4292 
Math. \& Sci. hum. / Mathematical Social Sciences (45e année, n 178, 2007(2), p. 119-124)

\title{
MATHÉMATIQUES, MUSIQUE ET ÉMOTION
}

\author{
Jean-Paul ALLOUCHE ${ }^{1}$, Laurence MAILLARD-TEYSSIER ${ }^{2}$
}

\author{
RÉSUMÉ - L'émotion ne serait-elle pas une différence irréductible entre mathématiques et \\ musique? \\ MOTS CLÉS - Émotion, Formalisation, Mathématiques, Musique \\ SUMMARY - Mathematics, music and emotion \\ An irreducible difference between mathematics and music might be emotion. \\ KEYWORDS - Emotion, Formalization, Mathematics, Music
}

\section{INTRODUCTION}

Lors de leur rencontre en décembre 1999 à l'IRCAM pour le Quatrième forum mathématique Diderot intitulé « Mathématiques et Musique, Logiques mathématiques, logiques musicales au XX $\mathrm{XX}^{\mathrm{e}}$ siècle, les deux auteurs de cet article - « officiellement » mathématiciens - prirent conscience dès leur première discussion que, contrairement à la quasi-totalité des mathématiciens présents, ils ne considéraient pas comme une évidence que les mathématiques et la musique aient des liens étroits. JPA avait coutume, un peu par provocation certes, de commencer ses conversations avec des compositeurs par l'affirmation qu'il n'y a pas de rapports entre mathématiques et musique, ou, en adoucissant cette assertion brutale, que les quelques rapports qui existent ne sont pas essentiels; LMT, plus nuancée dans son discours, suggéra une piste qu'ils n'allaient cesser de creuser dès lors : une différence irréductible entre mathématiques et musique est peut-être l'émotion. Cet article est une première tentative de synthèse des réflexions des deux auteurs sur ce sujet.

\section{UNE VIEILLE IDÉE PHILOSOPHIQUE DEVENUE UN PONCIF COMMODE}

Les philosophes grecs (citons bien sûr Pythagore et Platon) sont probablement à l'origine, en Occident, des rapprochements classiques entre mathématiques et musique et de manière plus générale entre «le Beau » et «les Nombres ». Leurs idées

\footnotetext{
${ }^{1}$ Laboratoire de Recherche en Informatique (LRI), CNRS, bâtiment 490, Université Paris-Sud, 91405 Orsay Cedex, allouche@lri.fr

${ }^{2}$ Faculté Léonard de Vinci SMBH (Santé-Médecine-Biologie humaine), Université Paris-Nord, 74 rue Marcel Cachin 93017 Bobigny Cedex, l.maillard@smbh.univ-paris13.fr
} 
(voisines d'ailleurs de pensées dans la Chine ancienne) ont été reprises et développées (citons au moins Leibniz : La musique est un exercice d'arithmétique secrète, et celui qui s'y livre ignore qu'il manie les nombres), mais elles ont été banalisées en des phrases toutes faites que l'on pourrait caricaturer en « Tout ce qui est nombre est beau » (sic), ou « Tout ce qui est beau est nombre » (sic), ou « Mathématiques et musique sont une seule et même activité »(sic), voire 《Les matheux sont musiciens $\gg($ sic $)$.

Il n'est pas inintéressant de tenter de creuser les deux dernières affirmations. Pour justifier la première, l'interlocuteur cite immédiatement les gammes : nous, nous contenterons de dire que c'est faire injure à la musique que de la réduire aux gammes, rythmes et autres quantifiables, un peu comme si on expliquait par exemple la Sonate pour flûte de Jolivet par le maillechort ou l'argent dont est fait l'instrument. Et d'ailleurs cette phrase affirmant l'identité des mathématiques et de la musique signifie-t-elle égalité, inclusion, ou plus modestement qu'il y a une intersection entre ces deux disciplines? Quant à la seconde affirmation, sans même demander une définition du matheux (enseignant? chercheur? intoxiqué au Sudoku?...) ou du musicien (mélomane? amateur jouant d'un instrument? compositeur?...), citons juste la géologue qui a un jour dit à JPA :

Ah bon, on dit ça chez les matheux? chez nous on dit que les géologues sont musiciens.

(Sur les mathématiques, les mathématiciens et leur sociologie, on lira avec délectation le livre de Didier Nordon [1981]).

Il n'est pas rare aussi d'entendre dire que les musiciens sont des mathématiciens, alors que - tout au plus - ils peuvent être des utilisateurs de concepts ou de techniques mathématiques souvent relativement simples.

\section{UNE AMORCE DE DISCUSSION SUR MATHÉMATIQUES ET MUSIQUE}

Les (non-)rapports entre musique et mathématiques, ou plus généralement entre arts et sciences ont suscité une immense littérature et nous n'avons ni la compétence, ni la place dans ce court article, pour en faire un survol exhaustif. Nous nous contenterons de donner deux débuts de pistes.

D'abord ce qu'on pourrait appeler comme F. Escal, « d'Alembert contre Rameau » [1983] et que nous résumerons par la citation suivante de d'Alembert empruntée à [Escal, 1983] :

N'imitons pas ces musiciens qui se croyant géomètres, ou ces géomètres qui se croyant musiciens, entassent dans leurs écrits chiffres sur chiffres, imaginant peut-être que cet appareil est nécessaire à l'art. L'envie de donner à leurs productions un faux air scientifique, n'en impose qu'aux ignorants et ne sert qu'à rendre leurs traités plus obscurs et moins instructifs.

On aura noté comme F. Escal que :

Le paradoxe en effet, c'est que c'est bien le géomètre d'Alembert qui va se révéler l'adversaire le plus radical des thèses physico-mathématiques avancées par Rameau dans ses écrits sur la musique. 
Une seconde piste est une phrase du compositeur (minimaliste) Tom Johnson : Ma musique ne doit pas éveiller d'émotion. On voit que la réflexion pourrait s'orienter vers l'opposition entre minimalisme et musique autobiographique comme dit T. Johnson, mais aussi entre disons musique baroque et musique romantique.

\section{PLAISIR OU/ET ÉMOTION?}

La pratique ou l'écoute de la musique peuvent susciter du plaisir. La pratique ou la lecture de mathématiques aussi. On lira en particulier le beau texte de J.-P. Kahane intitulé « Le plaisir des mathématiques »[2005]. Nous avons essayé d'expliquer ailleurs [Allouche, 1991] que le plaisir ressenti dans le second cas est plutôt un plaisir « linguistique », de reconnaissance de structure, qui nécessite une expertise a priori qui n'est pas toujours présente dans le cas d'une œuvre d'art, par exemple une ouvre d'une culture étrangère dont on n'a pas forcément les clés et qui peut susciter un plaisir, voire une émotion «bruts de fonderie ». Comme écrivait Anatole France : En art comme en amour l'instinct suffit.

Mais n'est-ce pas plus de plaisir que d'émotion qu'il s'agit dans le cas des mathématiques comme dans le cas d'un certain type d'écoute ou de pratique de la musique? Un plaisir lié à la reconnaissance d'archétypes, qui semble confirmer l'hypothèse de plaisir linguistique ou structurel que nous formulions plus haut. Ainsi Pascal Ceaux [2005], dans un article sur le 17 forum Le Monde-Le Mans (21 au 23 octobre 2005) sur le thème « La musique est-elle un art du penser? », parle-t-il des rapports entre la musique et le monde :

Quel rapport la musique entretient-elle avec le monde? L'universitaire André Charrak s'est interrogé sur cette vision issue de la tradition pythagoricienne d'une harmonie, d'un monde composé comme un concert. Ce schéma de pensée explique le plaisir que prend l'âme aux sons, car ils sont en accord avec des archétypes présents en elle, a-t-il expliqué. Le philosophe Spinoza avait réfuté cette position en la dénonçant comme un préjugé finaliste. Elle resurgit pourtant sous une nouvelle forme au XIX ${ }^{e}$ siècle par la voix du philosophe de la volonté Schopenhauer, pour qui la musique est la mélodie dont le monde est le texte.

\section{SPÉCIFICITÉ DE L'ÉMOTION MUSICALE}

Cher Mozart,

Tu me parles du monde d'où je viens.

Un monde d'avant le langage, un monde de pulsions et d'émotions, quelque chose qui se trouve sous le verbe. Tu me fais entendre la partition sentimentale de l'existence, [Schmitt, 2005].

L'émotion en musique nous semble d'une part non nécessairement liée aux caractéristiques quantifiables (mathématisables?) de la musique, d'autre part fondamentalement différente du ressenti (c'est à dessein que nous ne réutilisons pas le mot «émotion ») du mathématicien qui lit, invente (ou « découvre ») un joyau mathématique. 
Que l'émotion qui saisit l'auditeur, le compositeur ou l'interprète d'une pièce musicale ne soit pas directement liée à la structure et aux propriétés mathématiques de l'œuvre semble accepté, à condition toutefois comme plus haut de s'entendre sur le mot émotion (et l'absence d'émotion que demande T. Johnson nous semble une preuve a contrario de notre affirmation). Nous ne résistons pas à citer Kofi Annan, secrétaire général de l'ONU, dans son introduction à la conférence « De l'importance de la musique », le 8 novembre 2004 à New York, par L. Botstein :

Mais [la musique] est également mystérieuse. Le rythme et la tonalité peuvent être exprimés comme des formules mathématiques; les musiciens connaissent les techniques par lesquelles ils produisent un certain son. Mais aucun scientifique ou musicien ne peut expliquer le pouvoir que la musique a sur nos émotions. C'est probablement ce qu'avait à l'esprit un autre auteur grec qui a dit que la chanson est un cadeau divin de Dieu.

Nous aurons peut-être plus de difficulté à essayer de convaincre (au moins les mathématiciens) que l'émotion musicale n'est pas de même nature que le ressenti mathématique. L'émotion, cette capacité à, étymologiquement, sortir de soi, peut se voir comme physique plus qu'intellectuelle, si tant est qu'on puisse proposer cette dichotomie. La réaction de plaisir ou de contentement intellectuel en mathématiques que nous avons qualifiée de linguistique plus haut est un éblouissement, mais un éblouissement devant une structure reconnue, construite ou découverte, et qui demande de posséder un imposant corpus de connaissances avant de pouvoir y accéder, un peu comme la sensation que ressent un spécialiste des échecs devant un « beau » coup (comme nous le suggérions dans [Allouche, 1991], il faut pour cela non seulement connaître les règles du jeu d'échecs mais encore être capable de suivre le raisonnement du joueur qui l'a conduit à ce choix). D'ailleurs nous avons parlé de l'émotion qui saisit l'auditeur, le compositeur ou l'interprète d'une pièce musicale : mais qui jouerait le rôle de l'interprète en mathématiques? L'auteur exposant ses résultats? Le conférencier exposant d'autres travaux que les siens? Et où est l'émotion du mathématicien qui fait un exposé, si l'on fait abstraction de celle que transmet le passionné, quel que soit l'objet de sa passion?

Il nous semble aussi que les différents degrés de l'émotion en musique reflètent une sorte de dialectique et de circulation entre une composante physique, presque « animale » et une composante intellectuelle, ce qui est décrit dans le livre de Denton [2005] comme le croisement d'un processus intellectuel et d'un choc perceptif ou sensoriel. Ce qui se passe alors est lié à l'histoire sensible de l'individu plus qu'à son expertise. La musique - un peu comme la vision du Grand Canyon (cf. [Denton, 2005]) - va éveiller ou réveiller des émotions, qui ne seront pas qu'une sorte de jouissance intellectuelle mais qui peuvent être de la joie, de la tristesse, de la peur, de la colère...

\section{CONCLUSION}

En tentant d'analyser nos réticences aux amalgames courants entre les mathématiques et la musique et en essayant de dégager l'idée que l'émotion baigne l'une et pas vraiment les autres, nous pourrions donner l'impression qu'il y a l'émotion d'un 
côté et la rationalité de l'autre. Mais cela veut-il nécessairement dire que nous opposons émotion et raison? Deux citations peuvent nous éclairer. L'une de T. Nathan [Clément, Nathan, 2005, p. 101] :

Pour ce qui me concerne, je ne parviens pas à opposer émotion et raison. Je suis persuadé que l'émotion est un raisonnement en accéléré

nous permet de penser qu'on peut peut-être privilégier l'émotion sans nécessairement choir dans l'irrationalité; la seconde d'A. R. Damasio [2001, p. 331-332] :

La perception des émotions paraît vraiment dépendre d'un système spécial comprenant de nombreuses composantes, qui est indissociable de la régulation biologique. La faculté de raisonnement semble vraiment dépendre de systèmes neuraux spécifiques, dont certains se trouvent desservir la perception des émotions. Ainsi, il semble bien qu'il existe un fil conducteur reliant, sur le plan anatomique et fonctionnel, la faculté de raisonnement à la perception des émotions et au corps. C'est comme s'il existait une passion fondant la raison, une pulsion prenant naissance dans la profondeur du cerveau, s'insinuant dans les autres niveaux du système nerveux, et se traduisant finalement par la perception d'une émotion ou par une influence non consciente orientant un processus de prise de décision. La raison, de sa forme pratique à sa forme théorique, se développe probablement sur la base de cette pulsion innée, par un procesus ressemblant à l'acquisition d'une compétence supérieure dans la pratique d'un art. Si vous n'avez pas l'incitation de la pulsion, vous n'acquerrez jamais la mâ̂trise de l'art. Mais si vous possédez cette pulsion, cela ne garantit pas automatiquement que vous deviendrez un maître

donne une approche physiologique qui permet peut-être de réconcilier le processus physique, presque animal, et le processus intellectuel que nous évoquions plus haut, tout en montrant leurs limites respectives? Ainsi, au-delà de notre thèse de l'émotion comme facteur discriminant entre musique et mathématiques, aurait-on, avec cette intersection dans la géographie du cerveau de la faculté de raisonnement et de la perception des émotions, un indice d'un lien cognitif qui traduirait peut-être la ressemblance entre les vertiges, vertige de temps, vertige d'espace, vertige d'infinitude, que l'on ressent dans certaines activités mentales humaines - et pourquoi pas en particulier en mathématiques et en musique.

Remerciements. Les auteurs remercient chaleureusement Michèle Audin pour ses commentaires sur ce texte. 


\section{BIBLIOGRAPHIE}

ALLOUCHE J.-P., «Plaisir des mathématiques », Journal de l'ATHÉLEC (Association des thérapeutes du langage et de la communication) 29, 1991, p. 4-6.

CEAUX P., « La musique entre affects et mode de réflexion », Le Monde, 28 octobre 2005.

ClÉment c., nathan t., Le Divan et le Grigri, Odile Jacob (poches), 2005.

Damasio A. R., L'erreur de Descartes, Odile Jacob, 2001. [Traduction : M. Blanc].

DENTON D., Les émotions primordiales et l'éveil de la conscience, Nouvelle Bibliothèque Scientifique, Paris, Flammarion, 2005.

ESCAL F., « Musique et science : d'Alembert contre Rameau », International Review of the Aesthetics and Sociology of Music 14, 1983, p. 167-190.

KAHANE J.-P., « Le plaisir des mathématiques », RMS, Revue de la filière Mathématique, $116^{\mathrm{e}}$ année, octobre $2005, \mathrm{n}^{\circ} 1$. http://www.rms-math.com/article.php3 ?id_article=751

ou http://smf.emath.fr/VieSociete/Rencontres/France-Finlande-2005/KahaneF.pdf

NORdOn D., Les mathématiques pures n'existent pas!, Actes Sud, 1981. [Rééd. augmentée, 1993. Illustrations de M. Mendès France].

SChmitt É.-E., Ma vie avec Mozart, Paris, Albin Michel, 2005. 